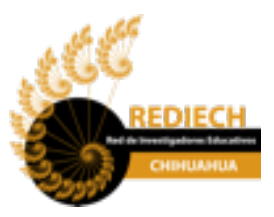

Red de Investigadores Educativos Chihuahua A.C. Chihuahua, México www.rediech.org

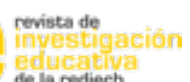

de la rediech

ISSN: 2007-4336

ISSN-e: 2448-8550

http://www.rediech.org/ojs/2017/index.php/ie rie rediech/index

Catherine Evelyn Araya Pérez

José Alejandro González Campos

2019

\title{
EVALUACIÓN DE LA POLÍTICA PÚBLICA EN CENTROS ESCOLARES SUSCRITOS A LA LEY DE SUBVENCIÓN ESCOLAR PREFERENCIAL
}

IE Revista de Investigación Educativa de la REDIECH, 10(19), pp. 275-297.

DOI: http://dx.doi.org/10.33010/ie_rie_rediech.v10i19.707

\section{(c) (i) (9)}

Esta obra está bajo licencia internacional Creative Commons Reconocimiento-NoComercial 4.0.

CC BY-NC 4.0 


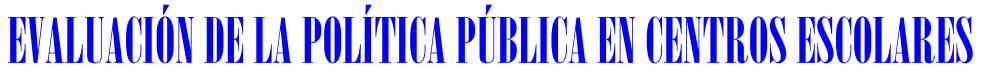

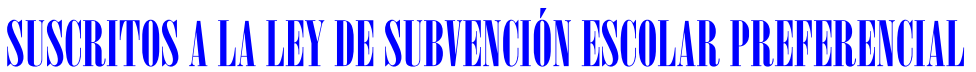

\author{
BIALUITIOI OF PIBLLC POLICY II SCIIOOLS SIBBSCRIBED \\ TO THE LAII OP PREAERETITILL SCHOOLLSIBSSIDY
}

\author{
ARAYA PÉREZ Catherine Evelyn \\ GONZÁLEZ CAMPOS José Alejandro
}

Recepción: julio 4 de 2019 | Aprobado para publicación: octubre 4 de 2019.

〜 C

DOI: http://dx.doi.org/10.33010/ie_rie_rediech.v10i19.707

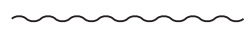

\section{Resumen}

La política pública chilena intenta reducir la brecha de resultados educativos entre escuelas municipales, particulares subvencionadas y privadas a través de un proceso llevado a cabo durante la última década. La Ley de Subvención Escolar Preferencial (SEP) ha sido parte de la solución del estado chileno a la problemática anterior. Esta investigación vincula predictores de la SEP con sus clasificaciones como variable respuesta. El objetivo es evaluar el impacto de la ley a través del análisis de las variables matrícula, resultados educativos e índices de vulnerabilidad, en contraste con la clasificación SEP asignada, utilizando un modelo estadístico cuantitativo. Las estadísticas de ocho años consecutivos

Catherine Evelyn Araya Pérez. Candidata a doctor del Programa de Doctorado en Políticas y Gestión Educativa de la Universidad de Playa Ancha, Chile. Magíster en Dirección y Liderazgo para la Gestión Educativa de la Universidad Andrés Bello. Su formación inicial es como profesora de castellano y actualmente coordinadora técnico-pedagógica de la Educación Pública en la ciudad de Valparaíso, con experiencia en la gestión directiva y técnico pedagógica en escuelas públicas y liceos humanistas científicos y técnico profesional. Su línea investigativa es la política pública educativa en el sistema escolar chileno y la gestión educativa en el sistema escolar. Correo electrónico: cataevy@ gmail.com. ID: https://orcid.org/0000-0003-2886-9421.

José Alejandro González Campos. Profesor de matemática y computación en la Universidad de Playa Ancha de Ciencias de la Educación, Chile. Es licenciado en Educación, magíster en Estadística en la Pontificia Universidad Católica de Valparaíso-Chile, doctor en Estadística en la Universidad Estadual de Campinas-Brasil. Coordinador General de Investigación Facultad de Ciencias Naturales y Exactas de la Universidad de Playa Ancha de Ciencias de la Educación. Coordinador de la Unidad de Soporte Estadístico para la Investigación de la Universidad de Playa Ancha. Coordinador del Laboratorio Experimental de Saberes Matemáticos de la Universidad de Playa Ancha. Coordinador de la asignatura metodología de investigación cuantitativa del Programa de Doctorado en Política y Gestión Educativa de la Universidad de Playa Ancha. Correo electrónico: jgonzalez@upla.cl. ID: https://orcid.org/0000-0003-4610-6874. 
(2008-2015) en la cohorte de cuarto básico de la región de Valparaíso fue el universo seleccionado para evaluar la consistencia entre la ley y la clasificación de las escuelas. La metodología del estudio es de tipo cuantitativa, longitudinal, explicativa y correlacional. Las técnicas de análisis de datos, descriptivas e inferenciales, establecen que los predictores estudiados, y considerados por la ley, no tuvieron un impacto significativo ni sostenido en el tiempo respecto de la clasificación, concluyendo una inconsistencia entre lo proyectado y lo observado. El impacto de la SEP no es la respuesta para equiparar los resultados.

\title{
Palabras clave: Ley de Subvención Escolar Preferencial, CLASIFICACIÓN SEP, RESULTADOS EDUCATIVOS, EVALUACIÓN DE LA LEY, CONSISTENCIA DE LA LEY.
}

\begin{abstract}
Chilean public policy tries to reduce the gap in educational level between municipal schools, subsidized and private schools, through a process carried out during the last decade. The Preferential School Grant Law (PSG) has been part of the Chilean state's solution to the aforementioned problem. This research links PSG predictors with their classifications as a variable answer. The objective is to evaluate the impact of the law, through the analysis of enrollment variables, educational results, and vulnerability indexes, in contrast to the assigned PSG classification, using a quantitative statistical model. Statistics of eight consecutive years (2008-2015) the cohort of Valparaíso region was the universe selected to assess the consistency between the law and the classification of schools. The study methodology is quantitative, longitudinal, explanatory and correlational. The data analysis techniques, descriptive and inferential, establish that the predictors studied, and considered by law, did not have a significant or sustained impact over time regarding the classification, concluding an inconsistency between the projected and the observed. The impact of the PSG is not the answer to bring the results to the same level.
\end{abstract}

Keywords: Preferential School Grant Law, SEP classification, EDUCATIONAL RESULTS, EVALUATION OF THE LAW, CONSISTENCY OF THE LAW.

\section{IrTRonecróín}

Según el informe para Chile PISA 2015, los resultados educativos que Chile obtuvo entre países pertenecientes a la Organización para la Cooperación y el Desarrollo Económicos (OCDE) se mantuvieron sin variaciones significativas durante

276 el periodo 2012-2014 (Levy y Schady, 2013). Estos resultados son coincidentes con 
los obtenidos en la prueba estandarizada que se aplica en Chile a través del Sistema de Medición de la Calidad Educativa (SIMCE) (Agencia de Calidad, 2012, 2013 y 2014; Gallegos, 2015). Ambos resultados representan un estado de la calidad de la educación y a su vez la influencia de las políticas públicas implementadas durante el periodo (Mizala y Torche, 2013).

Para este contexto (Rivas y Sánchez, 2016), la promulgación de marcos normativos específicos que fuesen en apoyo directo al desarrollo de la calidad de la educación chilena dieron paso al funcionamiento de las leyes que se declararon estar bajo los preceptos de un paradigma asociado a la discriminación positiva (Bouveau, 2005; Mineduc, 2009), como respuesta política para superar las fluctuaciones en los resultados educativos en general, y en particular la brecha existente entre los resultados de centros escolares de distintas dependencias administrativas.

De este modo, a partir de 2008, con la promulgación de la Ley 20.248, se propuso impulsar la calidad en el sistema educativo aplicando una fórmula de asignación de recursos económicos a escala diferenciada, dando especial tratamiento al estamento del sistema escolar con mayor índice de vulnerabilidad (IVE) de la población, reconociendo una importante concentración de estudiantes que presentaron este alto índice en los centros escolares de dependencia administrativa municipal, reforzando así el principio de equidad declarado tanto en la SEP (Mineduc, 2008) como en la Ley General de Educación.

Esta transformación sistémica y estructural dio paso a un cambio en la gestión escolar del sistema educativo a nivel nacional incorporando la suscripción de establecimientos escolares a convenios de igualdad de oportunidades y excelencia entre el Ministerio de Educación y los sostenedores: municipales o particulares subvencionados. La respuesta esperada en el mediano y largo plazo serían los resultados educativos asociados a las metas anuales y a los correspondientes ciclos de cuatro años, impactando de esta forma en la clasificación a las escuelas, acorde a la mejora educativa expresada en los resultados educativos SIMCE obtenidos, cuya concreción se encuentra en los planes de mejoramiento educativo (PME) como requisito de ingreso. Posteriormente, y luego de cada periodo anual y de ciclo, se instaló la exigencia de realizar tanto una evaluación de acuerdo a los niveles de impacto de las prácticas escolares instaladas y de los resultados educativos obtenidos en SIMCE como una rendición de cuentas anual de las acciones implementadas con recursos estatales, en coherencia a sus resultados de eficiencia interna.

En este contexto, la investigación permitirá determinar la consistencia de la implementación de la SEP durante el periodo 2008-2015 en las cohortes de cuarto básico, con respecto de sus estados de clasificación, en centros escolares de la región de Valparaíso que suscribieron a ella, determinando la(s) variable(s) predictora(s) responsable(s) de ello, cuantificando estadísticamente significancias, correlaciones y/o dependencias entre la SEP y el cambio de clasificación a través de un modelo explicativo que evalúa la Ley en su acción, siendo el modelo estadístico desde donde se propone una forma de evaluar para la toma de decisiones respecto de la política misma.

Las variables consideradas como predictoras incluidas en la investigación son: la matrícula, el índice de vulnerabilidad y los resultados educativos expresados en 
las pruebas estandarizadas de SIMCE. Se han seleccionado estas variables como predictoras por tres causas. La primera dice relación con la predominancia de estas tres variables en la normativa misma; a saber, los resultados SIMCE son la variable cuya asignación porcentual en la clasificación SEP de escuelas representa un 70\% del valor general. En segundo lugar, la variable índice de vulnerabilidad es aquella que genera el foco de la distribución de recursos, considerándose un factor determinante, pues releva el nivel socioeconómico (NSE) al cual pertenecen los estudiantes.

En cuanto a la matrícula, esta variable quedó asociada al registro de la asistencia diaria de los estudiantes a sus escuelas, determinando de esta forma el presupuesto inicial y anual de recursos SEP. Se esperaba que la matrícula creciera en los centros escolares públicos que iniciaban su adscripción a la SEP, producto del traspaso de recursos y de la gestión escolar bajo esta nueva institucionalidad.

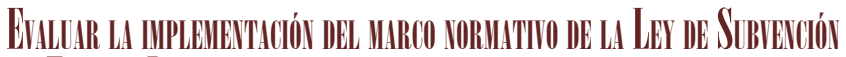

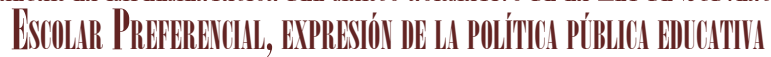

Los componentes estructurales de toda política pública, como la inversión presupuestaria, organización, comunicación, consecución de acciones, medios de verificación, indicadores de seguimiento u otros, representan un marco complejo, la cual durante su implementación va adoptando, representando y/o manteniendo los objetivos iniciales o modificándose según sea el análisis (Harguindéguy, 2015). El análisis evaluativo de la política pública educativa en Chile es un proceso técnico con una data de diez años, cuyos productos más comunes adoptan la forma de informes, dando paso a correcciones de tipo normativa, generalmente. Desde la promulgación de la Ley SEP hace ya 11 años se han generado a lo menos nueve modificaciones que inciden en su cuerpo legal, creando y/o afectándose otros marcos legales que se encuentran interrelacionados. Para la OCDE, el proceso de evaluación consiste en "determinar la pertinencia y el logro de los objetivos, así como la eficiencia, eficacia, impacto y sostenibilidad para el desarrollo" de una política vigente (OCDE, 2002, pp. 21-22). Esta investigación evalúa ocho años de implementación de la subvención escolar preferencial en centros escolares de la región de Valparaíso, con el fin de establecer su impacto en ellos a través de los resultados educativos y los datos secundarios de número de matrícula, índice de vulnerabilidad y resultados educativos del SIMCE obtenidos de bases de datos, cuyas cifras inciden en los cambios de clasificación. La hipótesis propuesta para este estudio es: la ley SEP afecta positiva y significativamente factores clave de la gestión escolar, asegurando la mejora de los centros escolares expresadas en sus clasificaciones.

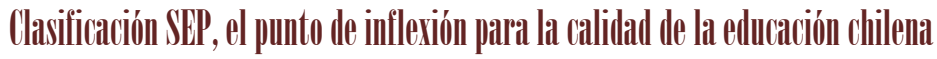

Desde el año 2000, Chile asume la obligación de dar muestras concretas de lo implementado en educación durante la década anterior, de tal modo que el tema de los resultados educativos ligados a la calidad ha pasado a convertirse en prioridad país 278 en materia de política educativa (Donoso-Díaz, De Souza y Gouveia, 2016). Los 
resultados obtenidos en pruebas estandarizadas como PISA, TIMMSS y otros test como TERCE y SERCE de UNESCO, durante la década del 2000, revelan algunos puntajes auspiciosos, por su incremento comparativo a través del tiempo; sin embargo, para otros fueron poco favorables, teniendo en cuenta que Chile presenta un coeficiente de Gini de 0.15 puntos porcentuales más alto que el reportado por los países de la OCDE (OCDE, 2018; Rivas y Sánchez, 2016).

Chile asume compromisos activos con el Banco Mundial y el Fondo Monetario Internacional, y entre las exigencias establecidas para evidenciar no solo el buen uso de los recursos, sino también el crecimiento del país como tal, se consideran los resultados educativos del SIMCE para evidenciar la calidad de la educación chilena. En el proceso de conducción hacia la consolidación de un sistema educativo que debe dar respuesta a los resultados esperados de un país OCDE, el rol activo lo tuvo y sigue teniendo una nueva institucionalidad creada para velar por la calidad: la Agencia de Calidad de la Educación, la cual se vincula directamente al Ministerio de Educación chileno (OCDE, 2016; OCDE, 2018).

En este periodo, y durante el primer lustro de implementación de la Ley SEP en Chile (2008-2013), las escuelas que se adscribieron a ella (Amaya, 2015) inician un proceso de permanente tensión en las comunidades escolares, involucrando sostenedores y marcos legales dispuestos para la mejora (Raczynski et al., 2013). El convenio firmado entre el Ministerio de Educación y sostenedores se encuentra enlazado al Sistema de Aseguramiento de la Calidad de la Educación (Mineduc, 2011), cuyo cuerpo legal determina requerimientos sobre la base de resultados e indicadores de evaluación que se hacen presentes en los estándares indicativos de desempeño (Mineduc, 2014).

En este contexto, la rendición de cuentas, como proceso extrapolado desde la empresa privada, se instala bajo la condicionante de la entrega de fondos presupuestarios a los sostenedores y por ende a sus estudiantes, emulando restricciones que otros países como Inglaterra, Francia y Estados Unidos habían instalado en sus propias políticas para generar regulación frente a la asignación de recursos fiscales. Esta política exigió instalar una cultura de responsabilización de la utilización de recursos asociados a la Ley SEP (Weinstein, Fuenzalida y Muñoz, 2012), utilizando como órgano rector y fiscalizador a la Superintendencia de Educación y la Contraloría General de la República, ambas institucionalidades cuyo rol fundamental es la supervisión.

Así, las indicaciones del Sistema de Aseguramiento de la Calidad de la Educación son claras al definir procesos de clasificación asociados a los resultados educativos SIMCE obtenidos, generando incentivos a aquellos establecimientos que construyen una trayectoria positiva (es decir, altos resultados evidenciados en el SIMCE) y sanciones en aquellos casos en que los resultados no sean los esperados, pudiendo llegar hasta la clausura de centros escolares que se mantienen por cuatro años consecutivos en la clasificación de en recuperación. La rendición de cuentas tiene su respaldo en indicaciones provenientes desde la OCDE y el Banco Mundial, las que se vinculan con la instalación de procesos de medición estandarizados, considerada como la única fuente verídica y estadísticamente válida para recoger antecedentes cuantitativos que dan cuenta de la calidad de los aprendizajes de los estudiantes 
en cuarto y octavo básico, y luego en segundo medio (Parcerisa y Falabella, 2017; Rivas y Sánchez, 2016).

\author{
Lospredictores
}

\title{
Índice de vulneriatililidid
}

El índice de vulnerabilidad, o IVE, se relaciona con el tipo de desventaja (Hernández, Cardona y Segura, 2018) asociada a una carencia de un capital social, humano, financiero o productivo, según Kaztman (Hernández, Cardona y Segura, 2018). Cada uno de ellos tiene subindicadores que lo componen y definen, complementando el significado complejo y multidimensional de la vulnerabilidad social. El Ministerio de Desarrollo Social chileno también ha hecho su aporte a la definición del concepto. En línea con el Banco Mundial, establece que la vulnerabilidad está asociada a situaciones de riesgo (Ministerio de Desarrollo Social, 2018) a las que se encuentran expuestas las familias. El IVE, asociado a educación, se acerca más al hecho de estar privado de acceder a la misma escuela y a recursos educativos complementarios, tecnológicos, culturales o profesionales que colaboran en el aprendizaje de los estudiantes, poniendo en riesgo la posibilidad de ascender socialmente, producto de este bien común (Brunner y Ganga, 2017). La organización de la Junta Nacional de Auxilio Escolar y Becas chilena (JUNAEB) también ha configurado una definición para el IVE, la cual se acerca más a un indicador de riesgo construido con base en diversos criterios como: ingresos, contexto social, contexto escolar y salud. A su vez, cada uno de ellos posee una descomposición que complejiza la conceptualización (Ñanculeo y Merino 2016). Chile ha considerado este concepto para relevar las necesidades situadas desde los estudiantes y establecer cuáles son las brechas necesarias de disminuir.

\section{Miatrículla y subrención esscolart}

En Chile, por 25 años la subvención o aporte presupuestario estatal por estudiante tuvo un mismo valor, independiente del nivel socioeconómico (NSE) o IVE que tuviese el estudiante, de su localidad de origen o la calidad del sostenedor: municipal o particular subvencionado (Mizala y Torche, 2013); e incluso del porcentaje de concentración de estudiantes con el mismo NSE existente, de alguna necesidad educativa especial (NEE); es decir, dificultades en el aprendizaje. Este sistema de asignación presupuestaria exige la presencia efectiva de los estudiantes en clases y penaliza la ausencia con la disminución de la subvención en el presupuesto correspondiente a cada centro educativo durante un mismo año lectivo. Durante el periodo en estudio, este sistema de subvención tuvo una modificación e incremento importante a partir de la entrada en vigencia de la Ley 20.408 (SEP), la cual por primera vez triplicará el valor de la unidad de subvención (USE), según el NSE que el estudiante tenga 0 la concentración del número de estudiantes con un bajo NSE en un mismo centro escolar (Weinstein, Fuenzalida y Muñoz, 2010), impulsando una nueva forma de buscar la equidad y calidad, especialmente en los centros educativos con más bajos resultados y más amplia brecha educativa. Este predictor toma relevancia al considerar que los centros escolares recibirán esta USE-SEP proporcionalmente a su declara280 ción de asistencia. La SEP abrió la oportunidad de discriminar positivamente entre 
los establecimientos con baja clasificación y resultados, generando expectativas de logro y calidad, activando la posibilidad de atraer matrícula hacia el sistema de mayor vulnerabilidad: el sector de administración municipal. Aun así, se ha presentado una suerte de paradoja creada por las propias condiciones de asignación de recursos. Los centros con más matrícula y concentración de estudiantes vulnerables reciben más recursos que aquellos que, en igual condición de vulnerabilidad y número de estudiantes en concentración, tienen menos estudiantes matriculados, disminuyendo considerablemente los recursos asignados.

\section{Ressultialdos eduluativos}

El testeo estandarizado abrió un espacio que igualó puntajes obtenidos en SIMCE con calidad educativa (Hernández y Raczynski, 2015; Pino, Oyarzún y Salinas, 2016). El SIMCE forma parte clave del engranaje que compone la normativa SEP, la cual incorpora entre sus formas de seguimiento el desempeño escolar, asignando un valor importante a los resultados SIMCE con respecto a la clasificación de los centros educativos, valorizados en un $70 \%$ del total de los indicadores que se toman en cuenta, siendo el $30 \%$ complementario obtenido desde el sistema de evaluación de desempeño o SNED (DIPRES, 2009), transformándose en una variable significativa en esta fórmula. La conexión entre SIMCE y rendición de cuentas exigida por la SEP es directa, con resultados más altos; la autonomía es un estado de clasificación viable, así como la liberación de recursos y administración delegada de estos. Su contraparte, resultados bajos en forma consecutiva, significará acercarse cada vez más a la opción del cierre del establecimiento.

\section{Veтononotí|}

La siguiente sección dice relación con la metodología adoptada en esta investigación, en la cual se presentará el objetivo de investigación, la unidad de análisis, las técnicas estadísticas desarrolladas y los resultados obtenidos en ella. La investigación se cierra con las conclusiones del estudio.

\section{Tipo de investigatción}

El estudio es del tipo cuantitativo, cuyo enfoque ha sido témporo longitudinal, con componentes descriptivos e inferenciales y de alcance correlacional (Hernández, Fernández y Baptista, 2014).

\section{Objectivo}

El objetivo de este estudio es evaluar el impacto de la Ley 20.408 SEP en un periodo de ocho años consecutivos a través de un modelo estadístico cuantitativo que describe y explica la consistencia de la SEP, considerando las variables de matrícula por cohorte, índice de vulnerabilidad, resultado SIMCE y clasificación SEP en escuelas y liceos estudiados de la región de Valparaíso durante el periodo 2008-2015. 


\section{Técniciass de recolección y antililisis de datos}

La recolección de los datos se hizo desde fuentes de información secundaria, tales como las bases de datos abiertos del Centro de Estudios del Ministerio de Educación de Chile para el periodo 2008-2015 y bases de datos abiertos de la Junta de Auxilio Escolar y Becas (Junaeb). También se revisaron los informes técnicos SIMCE y de desempeño de la Agencia de la Calidad de la Educación, junto a estadísticas internacionales de la OCDE referidas a resultados PISA. De las nacionales se construyó una base de datos exclusiva y de elaboración propia para este estudio, en la cual se reunieron piezas de información solo del periodo 2008-2015 de los establecimientos reunidos geográficamente en la región de Valparaíso, con 38 comunas revisadas para cada variable. La decisión tomada respecto del periodo temporal de estudio se basó considerado la entrada en vigencia de la Ley 20.408 y el último año en que se utilizó el proceso de clasificación de centros escolares.

A partir del año 2016, el Ministerio de Educación chileno introduce un cambio en el sistema de clasificación, emigrando desde la clasificación hacia las categorías de desempeño, el cual se asigna calculando los resultados educativos, o SIMCE, y nuevos indicadores, los cuales podrán ser considerados en futuras investigaciones con fines comparativos u otros. El análisis de datos se realizó a través de un proceso secuencial: una primera etapa descriptiva, en la cual se presenta el comportamiento de cada una de las variables en estudio, recurriendo a la exposición de medidas de posición (media y mediana), medidas de variabilidad (desviación estándar) y medidas de forma (curtosis y asimetría), visualizando cómo se expresa el nivel de impacto de las variables en los resultados de las unidades de análisis. Y una segunda etapa inferencial, o correlacional, en donde se aplicaron pruebas de asociación o dependencia entre las variables predictoras y la variable respuesta, cuyo criterio de decisión fue el $p$-valor en un nivel de confianza de un $95 \%(\alpha=0.05)$. Las técnicas a utilizar dependieron del estatus métrico de las variables que se sometieron a correlación (Hernández, Fernández y Baptista, 2014).

Con el fin de dar consistencia a las unidades muestrales y permitir el desarrollo de un estudio inferencial, el universo lo constituyeron las 4,725 escuelas y liceos adscritos a la Ley SEP en la región geográfica de Valparaíso durante el periodo 20082015 de 38 comunas, desde donde se obtuvo la unidad de análisis e información. De este universo se utilizó una muestra probabilística aleatoria, estratificada por cohortes. En ella se incluyeron a 1,863 establecimientos educacionales del universo inicial señalado, considerando el total de los ocho años de estudio, cuyo cálculo se obtuvo de la utilización de la fórmula de Namakforoosh (2000), con un nivel de confianza del $95 \%$ y un error de estimación del $5 \%(\alpha=0.5)$.

A su vez, se realizó una tercera etapa definitoria para la muestra de análisis, filtrando aquellos centros escolares que cuentan con un mínimo de cuatro años de información requerida, quedando constituida finalmente por 1,264 centros para el periodo de estudio. Para el correcto procesamiento de los datos se utilizaron el software R 3.5.1 y Jamovi 1.0.1, a través de los cuales se crearon los gráficos y tablas de apoyo y complemento en cada etapa del análisis. En función de la dinámica de 282 los predictores significativos, los resultados de las pruebas aplicadas revelarán tres 
escenarios posibles, a saber: 1) la inconsistencia interna, si los predictores de la Ley 20.248 no fuesen significativos; 2) la relatividad del impacto, si solo un porcentaje relativo de los predictores fuese significativo; y, 3) la consistencia interna, si todos los predictores fuesen significativos.

\section{VII modelo estadilistico culintitititio}

La fórmula (1) representa el modelo con el cual realizaremos el proceso de evaluación, donde $Y$ representa la variable respuesta, que son los estados de clasificación SEP en sus tres estados, a saber: autónomos, emergentes y en recuperación. Es importante señalar que existe un cuarto estado que no entra en la clasificación, pero que incide directamente en la selección de la muestra; este estado es la no clasificación, determinando el número de centros educativos que participan de esta muestra. Las demás variables se representan como $X$; constituyen cada una de las variables predictoras seleccionadas para este estudio y cada uno de las betas, los parámetros o pesos de cada predictor, los cuales serán estimados a partir de la muestra observada. Formalmente, esta relación de dependencia entre los predictores y respuesta, evidenciando los pesos, es representado por el siguiente modelo estadístico:

$$
Y=\beta_{0}+X_{1} \beta_{1}+X_{2} \beta_{2}+X_{3} \beta_{3}+\cdots+\varepsilon
$$

\section{Resiltinos}

En adelante se presentan los resultados de la etapa de análisis descriptivo e inferencial, los cuales irán siendo desarrollados según respondan las preguntas de investigación trazadas al inicio de la investigación. La secuencia de tablas creadas busca apoyar desde otra forma de representación los análisis realizados, y los gráficos representan secuencias temporales de datos que nos indicarán los resultados obtenidos luego de las pruebas.

\section{Btapi descriptivi}

En esta primera etapa inicial se analizaron las medias obtenidas de las clasificaciones SEP que las escuelas analizadas obtuvieron durante los ocho años estudiados (2008 -2015). Las medias aplicadas a los estados de la variable respuesta clasificación SEP permitieron establecer que no hubo cambios importantes en estos ocho años en estudio; es decir, los centros escolares mantuvieron preferentemente la clasificación emergente, concentrando el mayor porcentaje de escuelas en esta clasificación. No se producen cambios de clasificación en un porcentaje importante de centros escolares en ninguno de los años investigados; es decir, se mantiene la clasificación SEP durante todo este tiempo de análisis.

El gráfico 1 muestra el comportamiento en el tiempo de la clasificación SEP. Los datos analizados anualmente facilitan la descripción, donde cada número representa un estado de clasificación, siendo el número 1 el estado de clasificación 
en recuperación, el número 2 el emergente y el número 3 el autónomo. Este último es el estado que refleja el mayor desarrollo y desde allí el vínculo surge con la calidad de la educación impartida en esos centros escolares. El gráfico muestra que la media anual entre el año 2008 al 2010 en términos de clasificación SEP no supera el valor 1.5, reflejando que la totalidad de las escuelas no alcanzan en plenitud el estado emergente habiendo transcurrido cuatro años desde la implementación de la Ley 20.248. Mientras que en 2011 y 2012 se produce una disminución notoria en la clasificación que se acerca más al primer nivel de desarrollo: en recuperación, que al segundo: emergente. Estos dos años son principalmente preocupantes en términos de clasificación. Por último, en 2013 comienza nuevamente un repunte de clasificación hasta alcanzar solo en 2015 el estado de clasificación emergente después de ocho años de ejecución. Una escuela en calidad emergente aún es una escuela con deficiencias en la calidad de educación que se imparte, de acuerdo al significado y peso porcentual que se les asignan a los resultados educativos. En ellos podremos encontrar algunas explicaciones en la medida que las pruebas de análisis se desarrollen. Del mismo modo, la tabla 1 complementa los resultados para esta etapa, presentando los antecedentes que los análisis arrojaron de tal modo que respaldan las aseveraciones anteriores.

El seguimiento a la variable IVE también se ha considerado en esta etapa descriptiva. El gráfico 2 muestra el promedio anual y a su vez longitudinal de la variable índice de vulnerabilidad, presentando un incremento importante en un periodo de tiempo de ocho años consecutivos. En los años 2009 y 2010, el IVE no presenta incremento, sino más bien se mantiene y presenta una pequeña baja entre el año 2010 y 2011, año en que se inicia un punto de inflexión para asumir un aumento continuo desde el 2012 al 2015. Las razones de la disminución en los años 2010 y 2011 pueden deberse a factores de deserción del sistema escolar o al descenso en la matrícula de acuerdo con los indicadores demográficos.

El gráfico 3 presenta las medias obtenidas después de aplicar técnicas descriptivas correspondientes. Se aprecian las medias anuales y a su vez la trayectoria longitudinal de los resultados educativos para SIMCE lenguaje. En este gráfico se observan resultados fluctuantes que no superan los 200 puntos entre 2008 y 2014. Solo en 2015 es posible evidenciar un alza en los resultados por sobre los 200 puntos, aún muy por debajo del mínimo expresado en la normativa misma (250 puntos) para optar a un cambio en el estado de la clasificación desde emergente a autónomo (Mineduc, 2011).

\section{Btiapi inferencicial}

En esta segunda etapa se relacionó cada uno de los predictores analizados con la variable respuesta, partiendo por el gráfico 4, que muestra el resultado de las dependencias y correlaciones entre el índice de vulnerabilidad en relación a la clasificación SEP obtenida consecutivamente desde el 2008 al 2015. Basados en el coeficiente de correlación, entre 2008 y 2009 es posible observar que mientras aumenta el IVE, cambia la clasificación SEP, actuando independientemente. Entre 2010 y 2012 se produce una relación inversa, siendo este último año el punto más crítico, una relación

284 máxima que no supera el $8 \%$. Entre 2013 y 2015 se produce el mismo fenómeno 


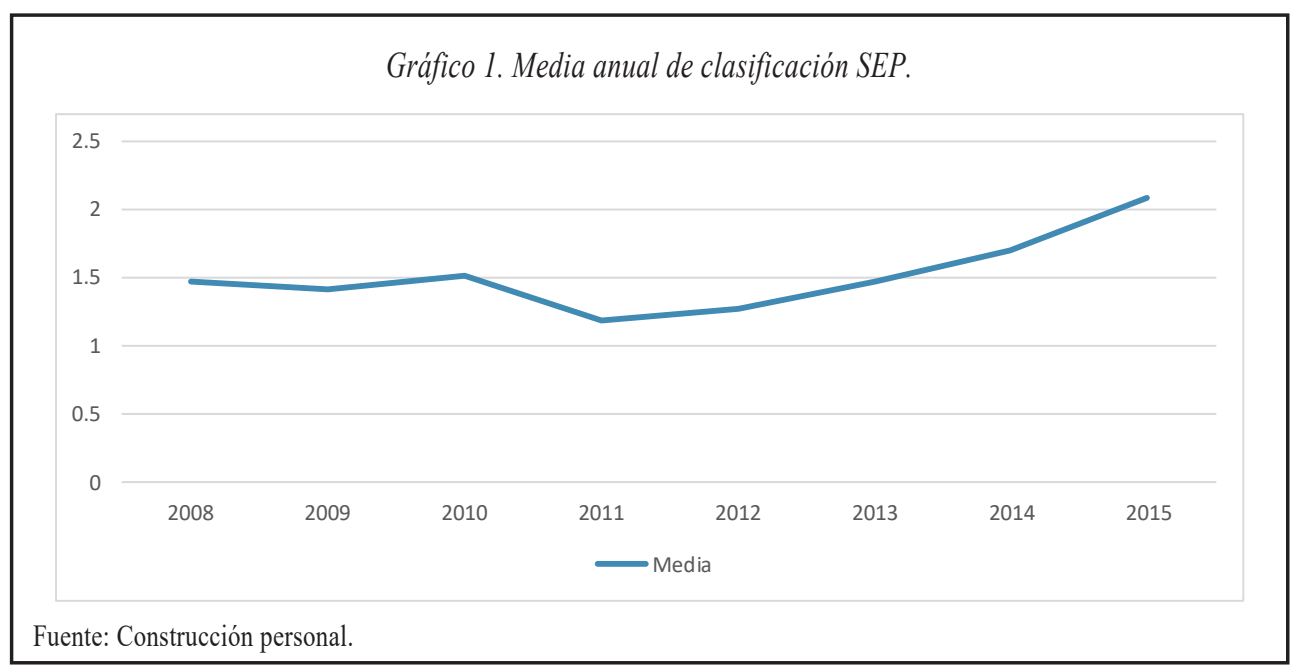

Tabla 1. Resumen de medianas de clasificación SEP en secuencia longitudinal (2008-2015)

\begin{tabular}{lcccccccc}
\hline & \multicolumn{7}{c}{ Clasificación SEP } \\
\cline { 2 - 9 } & $\mathbf{2 0 0 8}$ & $\mathbf{2 0 0 9}$ & $\mathbf{2 0 1 0}$ & $\mathbf{2 0 1 1}$ & $\mathbf{2 0 1 2}$ & $\mathbf{2 0 1 3}$ & $\mathbf{2 0 1 4}$ & $\mathbf{2 0 1 5}$ \\
\hline Mediana & 2,00 & 2,00 & 2,00 & 2,00 & 2,00 & 2 & 2,00 & 2 \\
\hline $\begin{array}{l}\text { Desviación } \\
\text { estándar }\end{array}$ & 0,658 & 0,532 & 0,468 & 0,406 & 0,416 & 0,530 & 0,462 & 0,413 \\
\hline
\end{tabular}

Fuente: Construcción personal.

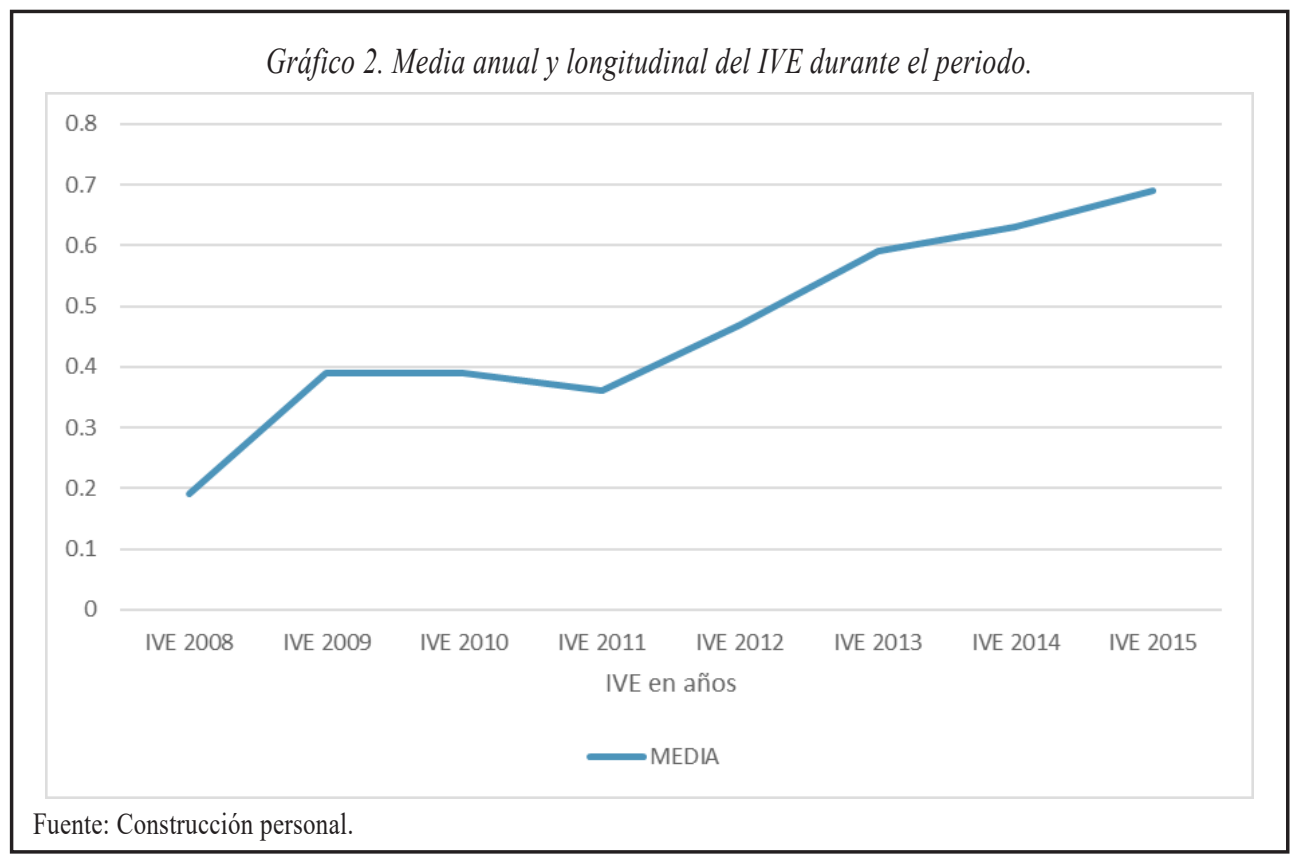


inicial de 2008 y 2009. Ante estos datos será un desafío futuro indagar respecto de cuáles fueron los factores que generaron la relación inversa entre 2010 y 2012.

Complementariamente al gráfico 4 de dependencias se incorpora la matriz de correlación entre el IVE y la clasificación SEP. En ella podemos establecer puntos de mayor significancia y por ende correlación, los que estarían en los años 2008, 2009, 2013, 2014 y 2015, quedando en forma atípica el año 2010 con un p-valor no significativo ( $\mathrm{p}$-valor $<0.005$ ) de esta correlación.

Con los mismos antecedentes fue posible aislar los centros educativos en clasificación emergente con distintos porcentajes de IVE. Entre 2010 y 2015 no hubo escuelas en clasificación emergente con IVE entre 40\% y 70\%. En 2009 hay un dato atípico, dentro del grupo de escuelas emergentes, un $38 \%$ de la muestra de escuelas están entre el 40\% y 70\% de IVE. Por otro lado, las escuelas con IVE sobre 70\%

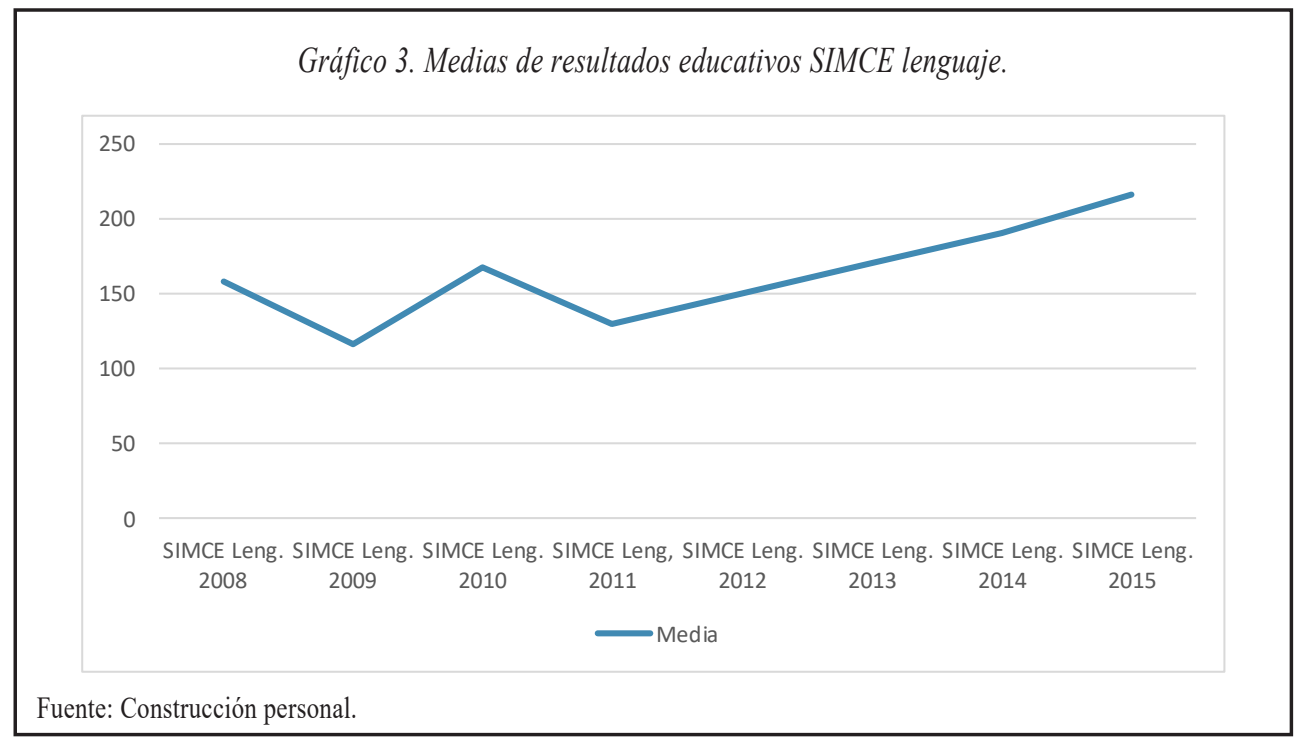

Gráfico 4. Secuencia temporal de dependencias entre IVE y clasificación SEP.

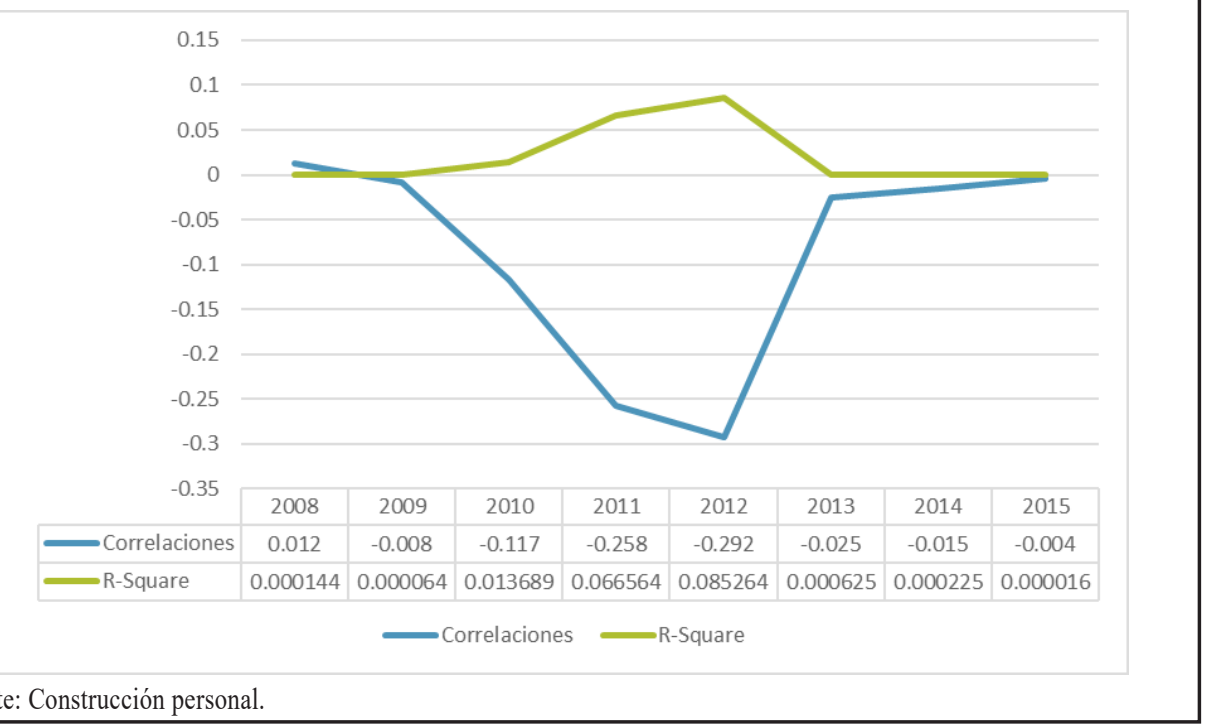




\begin{tabular}{|c|c|c|c|c|c|c|c|c|c|}
\hline \multirow[t]{2}{*}{ Clasif. } & & $\begin{array}{l}\text { IVE } \\
2008\end{array}$ & $\begin{array}{l}\text { IVE } \\
2009 \\
\end{array}$ & $\begin{array}{l}\text { IVE } \\
2010 \\
\end{array}$ & $\begin{array}{l}\text { IVE } \\
2011\end{array}$ & $\begin{array}{l}\text { IVE } \\
2012 \\
\end{array}$ & $\begin{array}{l}\text { IVE } \\
2013 \\
\end{array}$ & $\begin{array}{l}\text { IVE } \\
2014 \\
\end{array}$ & $\begin{array}{l}\text { IVE } \\
2015 \\
\end{array}$ \\
\hline & p-value & $<0.001$ & $<0.001$ & $<0.001$ & $<0.001$ & $<0.001$ & $<0.001$ & 0.637 & - \\
\hline \multirow{2}{*}{2008} & pearson's $r$ & 0.012 & 0.093 & -0.005 & -0.007 & -0.031 & -0.063 & -0.001 & -0.026 \\
\hline & p-value & 0.883 & 0.447 & 0.949 & 0.929 & 0.697 & 0.435 & 0.987 & 0.751 \\
\hline \multirow{2}{*}{2009} & pearson's $r$ & -0.074 & 0.008 & -0.050 & -0.045 & -0.063 & -0.071 & -0.019 & 0.011 \\
\hline & p-value & 0.360 & 0.948 & 0.458 & 0.579 & 0.430 & 0.374 & 0.815 & 0.894 \\
\hline \multirow{2}{*}{2010} & pearson's $r$ & 0.027 & -0.066 & -0.117 & -0.083 & -0.096 & -0.090 & -0.025 & -0.030 \\
\hline & p-value & 0.738 & 0.588 & 0.146 & 0.303 & 0.234 & 0.268 & 0.757 & 0.715 \\
\hline \multirow{2}{*}{2011} & pearson's $r$ & -0.301 & -0.265 & -0.290 & -0.258 & -0.293 & -0.306 & -0.040 & -0.182 \\
\hline & p-value & $<0.001$ & 0.028 & $<0.001$ & 0.001 & $<0.001$ & $<0.001$ & 0.620 & 0.025 \\
\hline \multirow{2}{*}{2012} & pearson's $\mathrm{r}$ & -0.336 & -0.077 & -0.278 & -0.259 & -0.292 & -0.329 & -0.027 & -0.243 \\
\hline & p-value & $<0.001$ & 0.530 & $<0.001$ & 0.001 & $<0.001$ & $<0.001$ & 0.739 & 0.002 \\
\hline \multirow{2}{*}{2013} & pearson's $r$ & 0.004 & -0.135 & -0.082 & -0.063 & -0.030 & -0.025 & -0.006 & 0.083 \\
\hline & p-value & 0.962 & 0.268 & 0.306 & 0.436 & 0.706 & 0.752 & 0.939 & 0.307 \\
\hline \multirow{2}{*}{2014} & pearson's $r$ & 0.114 & 0.028 & 0.002 & -0.005 & 0.004 & 0.021 & -0.015 & 0.050 \\
\hline & p-value & 0.160 & 0.818 & 0.979 & 0.947 & 0.957 & 0.792 & 0.852 & 0.542 \\
\hline \multirow{2}{*}{2015} & pearson's r & 0.116 & 0.108 & -0.010 & -0.004 & -0.003 & 0.011 & -0.016 & -0.004 \\
\hline & p-value & 0.157 & 0.379 & 0.903 & 0.957 & 0.974 & 0.893 & 0.842 & 0.966 \\
\hline
\end{tabular}

Fuente: Construcción personal.

en clasificación emergente representan el $66.6 \%$ de la muestra total, concluyendo que la mayor representación de escuelas en clasificación emergente se encuentra en establecimientos con un alto índice de vulnerabilidad; es decir, el hallazgo relevante es que a mayor IVE, la clasificación SEP no tiene variación. Otro antecedente importante de mencionar dice relación con que dentro del grupo de escuelas emergentes no existen escuelas con menos del $40 \%$ de vulnerabilidad. El porcentaje de escuelas emergentes que está entre $70 \%$ y más de IVE supera en un $28.6 \%$ el porcentaje de las que están entre el $40 \%$ y $70 \%$ de IVE. Se explica este fenómeno estadístico en atención al significativo porcentaje de centros escolares que se mantienen en la misma clasificación (emergentes) por un prolongado tiempo. Será motivo de un segundo desafío investigativo futuro profundizar en las causales de este fenómeno referido al hallazgo anterior: la relación a mayor IVE menor cambio de clasificación de modo ascendente. El gráfico 5 representa los antecedentes mencionados en este apartado.

Los cambios en la clasificación SEP en relación a los resultados educativos SIMCE lenguaje y matemática también fueron motivo de análisis en el modelo estadístico. De ellos podemos decir lo siguiente: tal y cual lo representa el gráfico 6, los resultados de SIMCE lenguaje en relación a la clasificación SEP, entre el 2009 y 2011, se visualiza un incremento en la dependencia que no supera el $6 \%$ de esta. Y a partir del 2012, las variables actúan de manera independiente. Se infiere que al predictor SIMCE lenguaje no es posible atribuirle una influencia significativa durante el periodo en estudio; en otras palabras, el hallazgo nos permite concluir que los resultados educativos SIMCE lenguaje no se explican necesariamente por la clasificación 

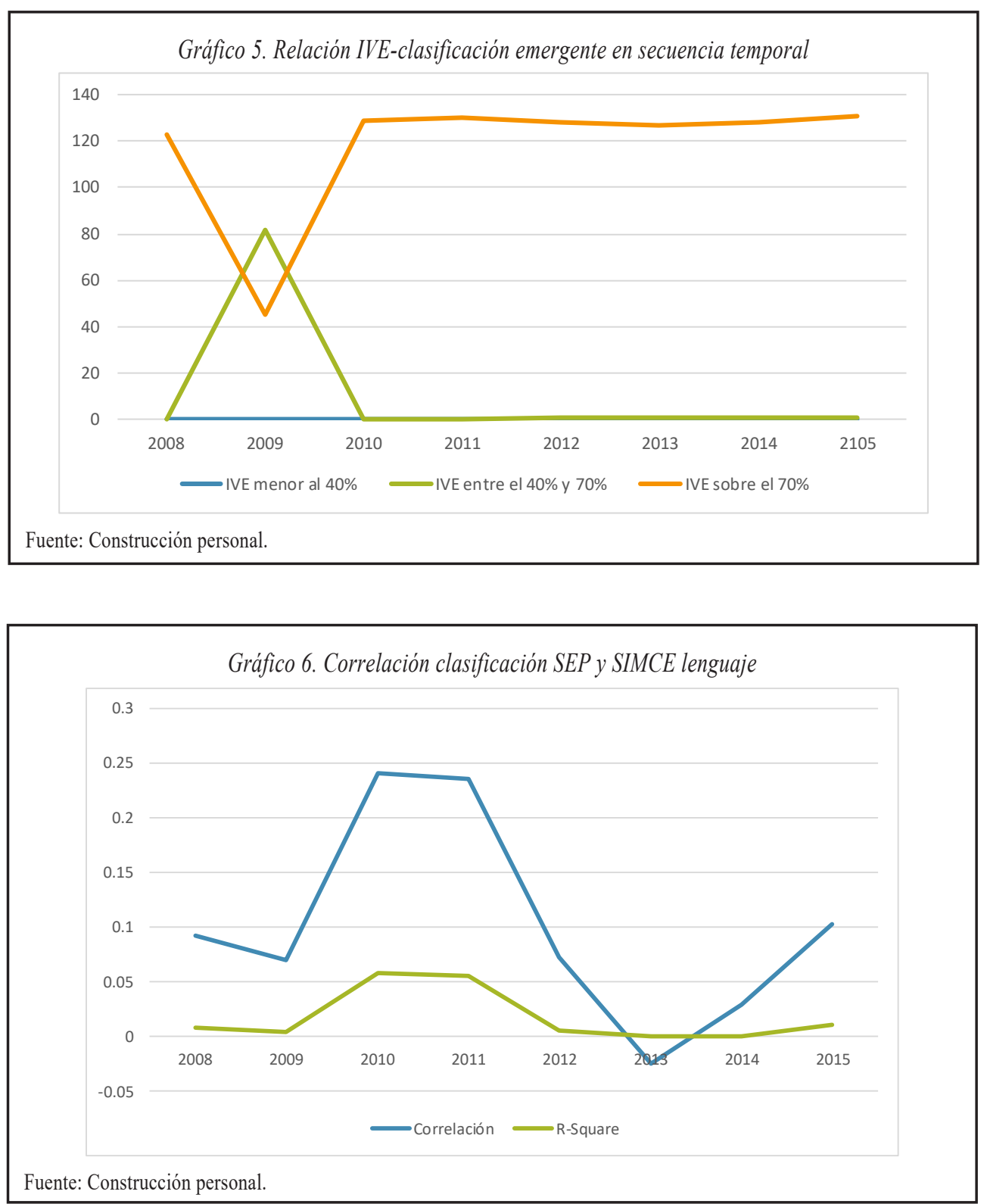

SEP que las escuelas poseen, alcanzando en este punto algunas coincidencias con el estudio realizado en la comuna de Punta Arenas (Venegas, Garay y Sillard, 2016).

Para complementar el gráfico 6 se incorpora la tabla 3, que contiene el análisis detallado realizado con la prueba inferenciales del coeficiente de correlación de Pearson, en la que se presenta la matriz de la correlación entre la clasificación SEP y los resultados educativos expresados en el SIMCE lenguaje, detallándose a través del p-valor con su respectivo grado de significancia existente entre ambas variables que sustentan los resultados obtenidos.

Del mismo modo, se buscó relacionar y establecer dependencias entre la clasificación SEP y los resultados educativos SIMCE matemática. El gráfico 7 muestra que 288 entre el año 2008 y 2010 existió un incremento en la correlación, aproximándose a 


\begin{tabular}{|c|c|c|c|c|c|c|c|c|c|}
\hline Clasif. & & $\begin{array}{c}\text { SIMCE } \\
\text { leng } \\
2008 \\
\end{array}$ & $\begin{array}{c}\text { SIMCE } \\
\text { leng } \\
2009 \\
\end{array}$ & $\begin{array}{c}\text { SIMCE } \\
\text { leng } \\
2010 \\
\end{array}$ & $\begin{array}{c}\text { SIMCE } \\
\text { leng } \\
2011 \\
\end{array}$ & $\begin{array}{c}\text { SIMCE } \\
\text { leng } \\
2012 \\
\end{array}$ & $\begin{array}{c}\text { SIMCE } \\
\text { leng } \\
2013 \\
\end{array}$ & $\begin{array}{c}\text { SIMCE } \\
\text { leng } \\
2014 \\
\end{array}$ & $\begin{array}{c}\text { SIMCE } \\
\text { leng } \\
2015 \\
\end{array}$ \\
\hline \multirow{2}{*}{2008} & pearson's r & -0.018 & -0.008 & -0.058 & -0.039 & -0.032 & -0.026 & -0.008 & -0.086 \\
\hline & p-value & 0.842 & 0.923 & 0.428 & 0.661 & 0.702 & 0.761 & 0.925 & 0.324 \\
\hline \multirow{2}{*}{2009} & pearson's r & 0.092 & 0.075 & 0.006 & 0.022 & -0.000 & 0.145 & 0.058 & 0.052 \\
\hline & p-value & 0.294 & 0.387 & 0.943 & 0.801 & 0.995 & 0.085 & 0.503 & 0.549 \\
\hline \multirow{2}{*}{2010} & pearson's r & 0.117 & 0.070 & 0.055 & 0.034 & 0.003 & 0.081 & 0.113 & 0.102 \\
\hline & p-value & 0.187 & 0.425 & 0.524 & 0.705 & 0.975 & 0.338 & 0.189 & 0.244 \\
\hline \multirow{2}{*}{2011} & pearson's r & 0.269 & 0.241 & 0.241 & 0.180 & 0.116 & 0.186 & 0.185 & 0.182 \\
\hline & p-value & 0.002 & 0.005 & 0.005 & 0.042 & 0.167 & 0.027 & 0.031 & 0.036 \\
\hline \multirow{2}{*}{2012} & pearson's r & 0.430 & 0.490 & 0.458 & 0.236 & 0.211 & 0.351 & 0.224 & 0.280 \\
\hline & p-value & $<0.001$ & $<0.001$ & $<0.001$ & 0.007 & 0.011 & $<0.001$ & 0.008 & 0.001 \\
\hline \multirow{2}{*}{2013} & pearson's r & 0.157 & 0.243 & 0.258 & 0.180 & 0.073 & 0.066 & 0.050 & 0.114 \\
\hline & p-value & 0.074 & 0.004 & 0.002 & 0.041 & 0.384 & 0.436 & 0.564 & 0.189 \\
\hline \multirow{2}{*}{2014} & pearson's r & 0.043 & 0.047 & 0.095 & 0.134 & 0.086 & -0.024 & 0.013 & 0.101 \\
\hline & p-value & 0.6431 & 0.586 & 0.272 & 0.132 & 0.309 & 0.776 & 0.882 & 0.247 \\
\hline \multirow{2}{*}{2015} & pearson's r & -0.000 & 0.023 & 0.081 & 0.140 & 0.088 & 0.012 & 0.029 & 0.103 \\
\hline & p-value & 0.998 & 0.793 & 0.354 & 0.117 & 0.301 & 0.885 & 0.741 & 0.238 \\
\hline
\end{tabular}

Fuente: Construcción personal.

un nivel de dependencia de un 9\% y de manera directa. Sin embargo, desde el 2011 hasta el 2013 las variables tienden a independizarse con un nivel de dependencia de $0.2 \%$, lo mismo que para 2015, año en que también vuelve a bajar. El año 2014, de manera atípica se incrementa en un $5 \%$ la correlación, fenómeno que representa un desafío para investigar tal atipicidad.

Del mismo modo que en la presentación de resultados educativos lenguaje, se incorpora la tabla número 4, que presenta el detalle de la correlación entre la clasificación SEP y los resultados educativos de matemática. La tabla 4 representa el p-valor de cada una de las correlaciones, indicándonos los puntos mayor y menor dependencia entre las variables.

La siguiente prueba estadística fue revisar la dependencia entre la variable respuesta: clasificación SEP y el predictor: matrícula. En esta revisión es posible establecer que entre los años 2008 y 2014, el porcentaje máximo de dependencia entre estas variables fue de un 4\%; es decir, prácticamente ambas variables son independientes entre sí. El gráfico 8 muestra la trayectoria del predictor, confirmando que no existe relación de dependencia entre ambas variables.

La tabla 5 nos presenta el detalle de la matriz de correlación obtenida luego de aplicar las pruebas de inferenciales coeficiente de Pearson para las variables de matrícula y clasificación SEP entre el 2008 y 2015. La matriz respalda lo explicado en el gráfico 8, estando los puntos de mayor significancia en los años 2012 y 2014; sin embargo, antes y después de estos años la relación existente entre ambas variables es totalmente independiente, al igual que su proyección hacia 2015.

La tabla 6 resume los valores de significancia dados por el p-valor de cada una de las variables predictoras analizadas en este estudio. A saber, índice de vulnera- 


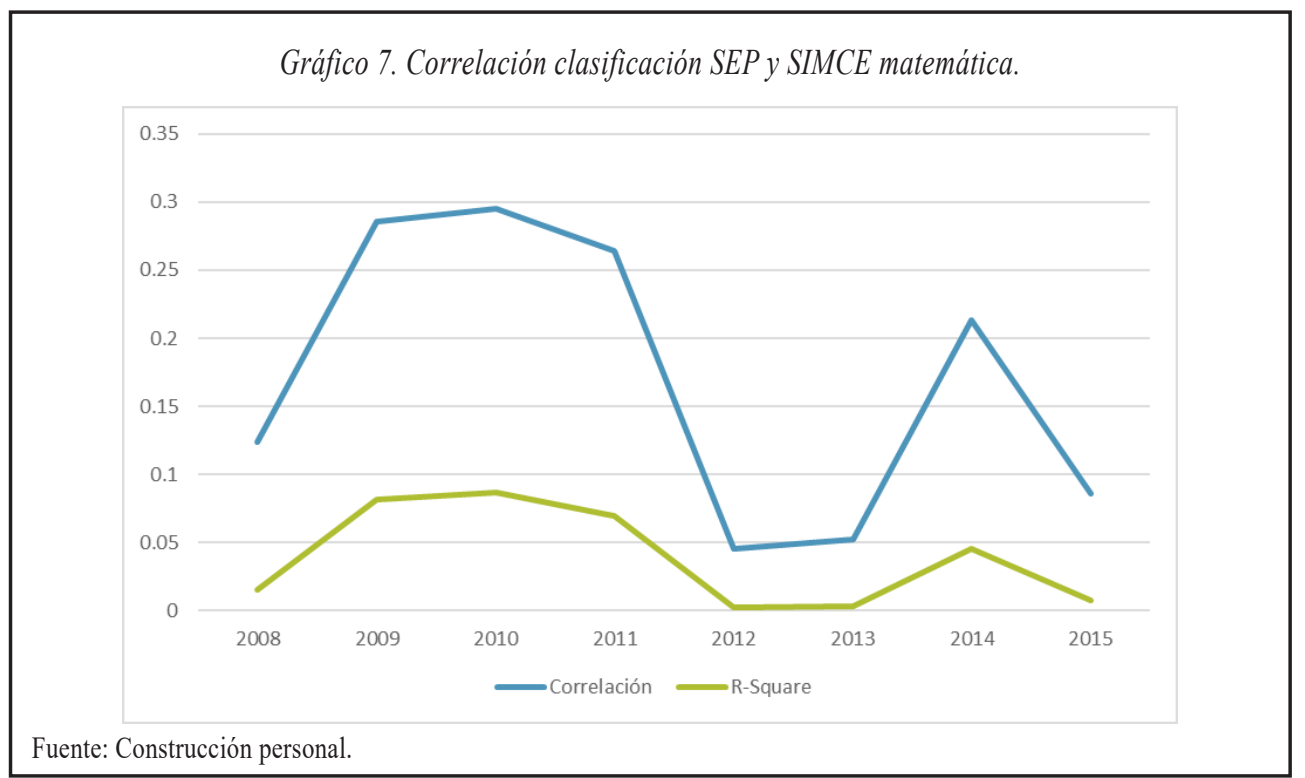

\begin{tabular}{|c|c|c|c|c|c|c|c|c|c|}
\hline \multirow{2}{*}{ Clasif. } & & \multicolumn{8}{|c|}{ SIMCE matemática } \\
\hline & & 2008 & 2009 & 2010 & 2011 & 2012 & 2013 & 2014 & 2015 \\
\hline \multirow{2}{*}{2008} & pearson's $\mathrm{r}$ & -0.044 & -0.124 & $0.234 * *$ & $0.313 * * *$ & $0.462 * * *$ & $0.279 * *$ & 0.110 & 0.057 \\
\hline & p-value & 0.619 & 0.157 & 0.007 & $<0.001$ & $<0.001$ & 0.001 & 0.213 & 0.518 \\
\hline \multirow{2}{*}{2009} & pearson's $\mathrm{r}$ & -0.007 & 0.098 & $0.286 * *$ & $0.285 * *$ & $0.505 * * *$ & $0.410 * * *$ & 0.107 & 0.110 \\
\hline & p-value & 0.944 & 0.304 & 0.002 & 0.002 & $<0.001$ & $<0.001$ & 0.267 & 0.255 \\
\hline \multirow{2}{*}{2010} & pearson's $r$ & -0.059 & 0.050 & 0.166 & $0.295 * * *$ & $0.489 * * *$ & $0.310 * * *$ & 0.160 & $0.189 *$ \\
\hline & p-value & 0.503 & 0.574 & 0.061 & $<0.001$ & $<0.001$ & $<0.001$ & 0.072 & 0.034 \\
\hline \multirow{2}{*}{2011} & pearson's $r$ & 0.051 & 0.103 & 0.089 & $0.198 *$ & $0.264 * *$ & $0.176^{*}$ & 0.140 & $0.175^{*}$ \\
\hline & p-value & 0.565 & 0.241 & 0.318 & 0.024 & 0.002 & 0.045 & 0.116 & 0.048 \\
\hline \multirow{2}{*}{2012} & pearson's $\mathrm{r}$ & -0.062 & 0.032 & 0.044 & 0.139 & $0.310 * * *$ & 0.045 & 0.100 & 0.139 \\
\hline & p-value & 0.456 & 0.699 & 0.606 & 0.098 & $<0.001$ & 0.590 & 0.237 & 0.101 \\
\hline \multirow{2}{*}{2013} & pearson's $r$ & 0.027 & 0.160 & 0.126 & $0.184 *$ & $0.318 * * *$ & 0.110 & 0.052 & 0.104 \\
\hline & p-value & 0.744 & 0.054 & 0.134 & 0.027 & $<0.001$ & 0.186 & 0.541 & 0.220 \\
\hline \multirow{2}{*}{2014} & pearson's $r$ & 0.024 & 0.140 & $0.282 * *$ & $0.225 * *$ & $0.215^{*}$ & 0.182 & 0.204* & $0.213 *$ \\
\hline & p-value & 0.783 & 0.104 & $<0.001$ & 0.009 & 0.012 & 0.033 & 0.017 & 0.013 \\
\hline \multirow{2}{*}{2015} & pearson's $r$ & -0.050 & 0.001 & 0.037 & 0.075 & $0.248 * *$ & 0.018 & 0.109 & 0.086 \\
\hline & p-value & 0.551 & 0.987 & 0.662 & 0.376 & 0.003 & 0.831 & 0.192 & 0.305 \\
\hline
\end{tabular}

$* \mathrm{p}<0.05 . * *<0.01 . * * * \mathrm{p}<0.001$.

Fuente: Construcción personal.

Tabla 6. Significancia de las variables predictoras con respecto a la clasificación SEP

\begin{tabular}{lcccccccc}
\hline & \multicolumn{8}{c}{ Clasificación SEP } \\
\cline { 2 - 10 } & $\mathbf{2 0 0 8}$ & $\mathbf{2 0 0 9}$ & $\mathbf{2 0 1 0}$ & $\mathbf{2 0 1 1}$ & $\mathbf{2 0 1 2}$ & $\mathbf{2 0 1 3}$ & $\mathbf{2 0 1 4}$ & $\mathbf{2 0 1 5}$ \\
\hline$R^{2}$ & 0.00227 & 0.0631 & 0.133 & 0.152 & 0.214 & 0.0145 & 0.0403 & 0.00131 \\
\hline \multicolumn{3}{l}{ Fuente: Construcción personal. }
\end{tabular}




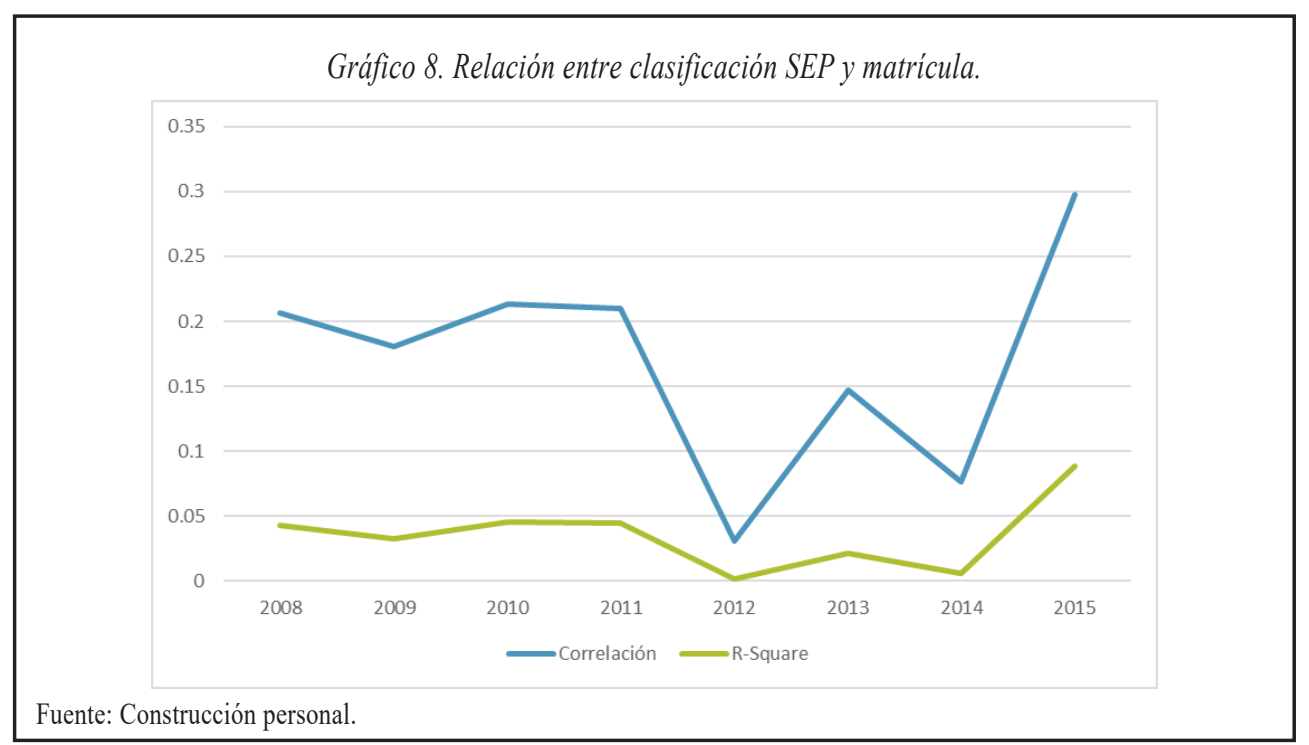

Tabla 5. Matriz de correlación clasificación SEP y matrícula (2008-2015)

\begin{tabular}{cccccccccc}
\hline \multirow{2}{*}{ Clasif. } & & \multicolumn{7}{c}{ Matrícula } \\
\cline { 3 - 10 } & $\mathbf{2 0 0 8}$ & $\mathbf{2 0 0 9}$ & $\mathbf{2 0 1 0}$ & $\mathbf{2 0 1 1}$ & $\mathbf{2 0 1 2}$ & $\mathbf{2 0 1 3}$ & $\mathbf{2 0 1 4}$ & $\mathbf{2 0 1 5}$ \\
\hline \multirow{2}{*}{2008} & pearson's r & 0.011 & -0.007 & -0.007 & -0.018 & -0.010 & -0.028 & -0.033 & -0.003 \\
\cline { 2 - 10 } & p-value & 0.892 & 0.933 & 0.926 & 0.0827 & 0.896 & 0.733 & 0.689 & 0.971 \\
\hline \multirow{2}{*}{2009} & pearson's r & 0.260 & 0.278 & 0.279 & 0.271 & 0.269 & 0.271 & 0.269 & 0.335 \\
\cline { 2 - 10 } & p-value & $<0.001$ & $<0.001$ & $<0.001$ & $<0.001$ & $<0.001$ & $<0.001$ & $<0.001$ & $<0.001$ \\
\hline \multirow{2}{*}{2010} & pearson's r & 0.268 & 0.265 & 0.264 & 0.256 & 0.255 & 0.256 & 0.244 & 0.242 \\
\cline { 2 - 10 } & p-value & $<0.001$ & $<0.001$ & $<0.001$ & 0.001 & 0.001 & 0.001 & 0.003 & 0.003 \\
\hline \multirow{2}{*}{2011} & pearson's r & 0.308 & 0.345 & 0.370 & 0.378 & 0.394 & 0.391 & 0.396 & 0.390 \\
\cline { 2 - 10 } & p-value & $<0.001$ & $<0.001$ & $<0.001$ & $<0.001$ & $<0.001$ & $<0.001$ & $<0.001$ & $<0.001$ \\
\hline \multirow{2}{*}{2012} & pearson's r & 0.282 & 0.282 & 0.296 & 0.305 & 0.312 & 0.308 & 0.315 & 0.329 \\
\cline { 2 - 10 } & p-value & $<0.001$ & $<0.001$ & $<0.001$ & $<0.001$ & $<0.001$ & $<0.001$ & $<0.001$ & 0.001 \\
\hline \multirow{2}{*}{2013} & pearson's r & 0.111 & 0.123 & 0.115 & 0.115 & 0.114 & 0.118 & 0.104 & 0.081 \\
\cline { 2 - 10 } & p-value & 0.169 & 0.125 & 0.154 & 0.153 & 0.158 & 0.142 & 0.200 & 0.322 \\
\hline \multirow{2}{*}{2014} & pearson's r & 0.119 & 0.122 & 0.083 & 0.067 & 0.072 & 0.061 & 0.057 & 0.092 \\
\cline { 2 - 10 } & p-value & 0.143 & 0.132 & 0.310 & 0.408 & 0.377 & 0.451 & 0.480 & 0.257 \\
\hline \multirow{2}{*}{2015} & pearson's r & 0.053 & 0.043 & 0.029 & 0.031 & 0.034 & 0.038 & 0.041 & 0.052 \\
\cline { 2 - 9 } & p-value & 0.518 & 0.601 & 0.727 & 0.709 & 0.677 & 0.645 & 0.616 & 0.523 \\
\hline
\end{tabular}

Fuente: Construcción personal.

bilidad, matrícula, resultados educativos SIMCE lenguaje y SIMCE matemática, en relación a la variable respuesta clasificación SEP. Los resultados de este análisis concluyen que para el año 2008 y 2009 no existe dependencia significativa entre los predictores declarados con la clasificación SEP ( $p>0.05)$. En el año 2010 es posible evidenciar un mínimo de dependencia, alcanzando un nivel de explicación de un $13 \%$ entre las variables predictoras y la variable respuesta: la clasificación SEP. Sin 
embargo, esta dependencia se debe particularmente a la matrícula ( $\mathrm{p}=0.001)$. Algo similar ocurre en el año 2011, donde se explica un $15 \%$ de la dependencia debido a la incidencia de la variable matrícula. En 2012, la significancia aumenta en un 21\% debido a las variables predictoras de mayor significancia, que fueron matrícula y el SIMCE matemática, para luego ir bajando otra vez en 2013 a un 14\%. En 2014 se produce un fenómeno atípico, donde la clasificación SEP está siendo explicada en un $40 \%$, cuya variable predictora de esta atipicidad correspondió a los resultados educativos de SIMCE matemática, particularmente. En 2015, vuelve a establecerse la dependencia de un $13 \%$ entre las variables predictoras y la variable respuesta sin que alguna de las variables incida en este porcentaje de dependencia. En general, se puede establecer una heterogeneidad de variables que afectan la clasificación, aunque todas en un bajo nivel de significancia, por lo que no es posible establecer una constante ascendente en la variable respuesta clasificación SEP, ni tampoco asegurar que existe una o más de una variable que afecta directamente este incremento.

Complementariamente a los análisis presentados, se integran gráficos que permiten revisar el índice de incremento en cada una de las variables. La medida de incremento se definió como una medida que visibiliza el crecimiento de la variable durante la trayectoria de investigación. Para resumir la dinámica de la clasificación SEP, IVE, matrícula y SIMCE, se confeccionó un índice de incremento que presenta la estabilidad, el descenso o aumento a lo largo del tiempo de cada una de estas variables, basado en la media de los incrementos anuales. La aparición de este índice de incremento ha sido un hallazgo relevante, pues intenta representar el trayecto temporal o comportamiento que ha tenido la variable durante el periodo de estudio. El gráfico 9 representa el índice de incremento de la clasificación SEP; en él se establece que durante los ocho años de estudio el $8 \%$ de la muestra presenta un índice de incremento negativo, solo un $36 \%$ de la muestra logra un incremento positivo y un $56 \%$ no presenta crecimiento alguno. Esta gráfica es de gran importancia y aporte al análisis y a los resultados, puesto que confirma que nuestra variable respuesta no está teniendo los cambios esperados, rechazando de este modo la hipótesis inicial y concluyendo que a pesar de las variables predictoras ninguna de ellas ha podido generar cambios en la clasificación que expresa la calidad de la cual se enuncia en la Ley de Subvención Escolar Preferencial, en la Ley de Aseguramiento de la Calidad y en la política pública para la educación chilena.

Respecto del gráfico 10, en él se establece el índice de incremento de la variable predictora IVE de la muestra, presentando hallazgos tan importantes como el anterior. El 94\% de la muestra presenta un incremento positivo en la condición de vulnerabilidad durante la trayectoria, solo el 3\% no presenta cambios y el otro $3 \%$ tiene un incremento negativo. La cifra de incremento positivo muestra un tema de fondo asociado a la condición social que declaran las familias durante el periodo en estudio, por lo que es posible señalar que la región en estudio se mantiene permanentemente en un estado de vulnerabilidad; asociado al nulo incremento de la clasificación SEP, genera un escenario educativo que relega las posibilidades de desarrollo en esta área. No será el IVE la variable predictora determinante en el cambio de clasificación SEP.

Los gráficos 11 y 12 muestran el índice de incremento de los resultados educati292 vos reflejados en los test estandarizados de SIMCE aplicados a la cohorte de cuarto 

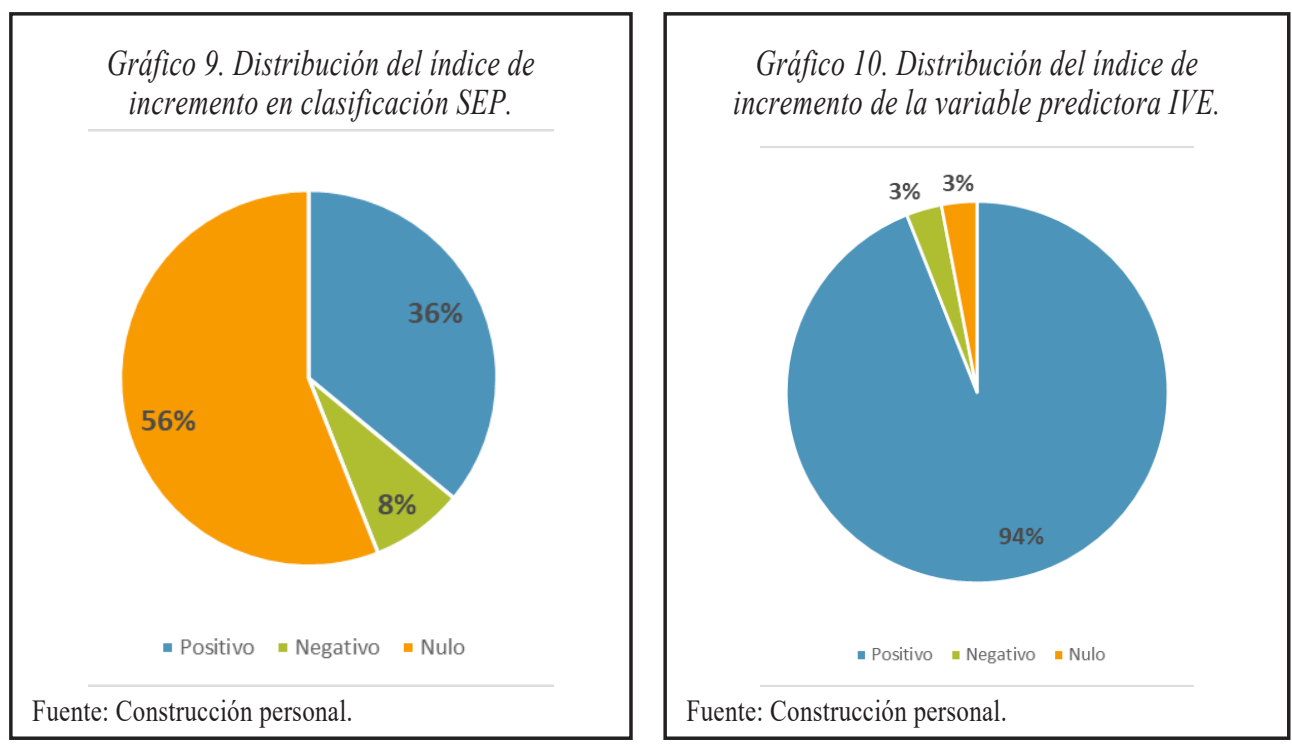

básico. En ambos índices es posible evidenciar hallazgos relevantes. El gráfico 11 representa el índice de incremento de SIMCE lenguaje, en el cual los hallazgos revelan similitud porcentual entre el incremento positivo y el nulo; es decir, durante los años en estudio 2008-2015, el incremento positivo no representa predominancia porcentual por sobre el incremento nulo y negativo. El $42 \%$ de incremento positivo resultado bajo para la amplia trayectoria temporal, las causales y consecuencias de este hecho podrán ser motivo de una investigación futura. A diferencia del gráfico 11, el 12 representa el índice de incremento en SIMCE matemática. En esta gráfica el incremento positivo se encuentra 9 puntos percentiles por sobre el mismo índice en SIMCE lenguaje, representando una mejora; pero esta mejora no logra superar significativamente el índice nulo y negativo juntos. En consecuencia, tampoco este predictor en su trayectoria representa mejora significativa en las escuelas de la región de Valparaíso.

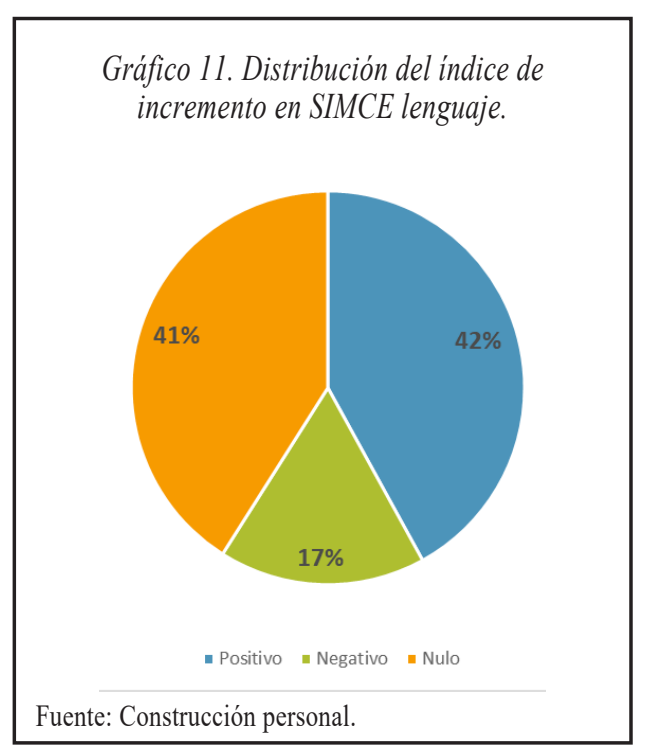

Gráfico 11. Distribución del indice de MCE lenguaje. 


\section{Conculsiones}

Respecto del objetivo de investigación: evaluar el impacto de la ley de subvención escolar preferencial a través del análisis de las variables matrícula, resultados educativos e índices de vulnerabilidad en contraste con la clasificación SEP asignada, se concluye que la clasificación SEP no tiene relación significativa $(\alpha=0.05)$ a partir de sus predictores para las escuelas de esta región. A partir de esta primera conclusión se evidencia que el impacto de la SEP no ha sido la respuesta para equiparar los resultados durante los ocho años en estudio en la región de Valparaíso.

Cada variable en estudio presenta un comportamiento independiente de la clasificación. No es posible atribuir el índice de incremento en la clasificación SEP a alguna de las variables en cuestión, pues en cada análisis se demostró que la correlación no fue significativa (p-valor $>0.05$ ).

El índice de incremento de la clasificación SEP porcentualmente no es importante, por lo que la acción de los soportes de la ley misma explica solo un $36 \%$ de la clasificación positiva, muy por debajo del incremento nulo.

Si el alto porcentaje del índice de incremento positivo del IVE (94\%) se asocia al bajo porcentaje del índice de incremento positivo de la clasificación SEP (36\%), se revela la importancia de revisar la política educativa referida al marco normativo de la SEP, y otros asociados en términos de su bajo impacto obtenido en el periodo en estudio para la región de Valparaíso investigada.

Al considerar las conclusiones del estudio realizado en la comuna de Punta Arenas para el mismo periodo de tiempo (Venegas, Garay y Sillard, 2016) descubrimos que estas apuntan a relacionar el IVE como un fuerte predictor del rendimiento académico y a sancionar que la ley SEP propiamente tal no ha mejorado los puntajes SIMCE ni ha revertido la baja de matrícula en esa comuna. En algunos aspectos, el presente estudio, trasladado a la región de Valparaíso, evidencia hallazgos respecto a que la clasificación asignada a los centros escolares a partir de su suscripción a la ley SEP, no se correlaciona en forma directa con ninguna de las tres variables en estudio. Es decir, no es posible determinar consistencia entre las tres variables predictoras (matrícula, resultados educativos SIMCE e IVE) en relación a la variable respuesta: clasificación. No obstante, hallazgos pormenorizados del análisis establecen un estancamiento de la clasificación SEP en los primeros seis años iniciales consecutivos de este estudio, ascendiendo lentamente entre el 2014 y 2015, sin superar la clasificación emergente.

En el estudio de Mizala y Torche (2013) se establece positivamente el alineamiento en la gestión escolar con los objetivos de la implementación de la Ley SEP en las escuelas, y además destaca la mejora de los resultados educativos a través de las mediciones estandarizadas. A diferencia de aquel estudio, y comprendiendo el alcance focalizado que esta investigación tuvo, este no coincide con aquellas conclusiones. Los hallazgos de esta investigación establecen que los centros educacionales suscritos a la SEP han incrementado su índice de vulnerabilidad, sin consistencia entre la clasificación SEP y los resultados educativos.

El estudio realizado por Carrasco, Pérez y Núñez (2015) realiza una interesante 294 comparación cuatro años antes del inicio de la implementación de la SEP con los 
resultados obtenidos cuatro años después (2004-2008/2009-2012). Los hallazgos fueron coincidentes con esta investigación, concluyendo que no hubo diferencias significativas en los resultados educativos SIMCE de lenguaje y matemática, y las diferencias que se encontraron entre estudiantes vulnerables y no vulnerables no son posible de atribuirse a la acción propia de la SEP. El presente estudio, sin realizar la diferencia entre estudiantes vulnerables y no vulnerables, establece el índice de incremento del IVE y lo correlaciona directamente con la clasificación SEP, obteniendo resultados similares a los de Carrasco, Pérez y Núñez. En la región de Valparaíso, los establecimientos suscritos a la Ley SEP no presentan un cambio significativo en ninguna de las variables analizadas. Los cambios que se presentaron fueron atípicos y no tienen relación con la clasificación SEP asignadas a las escuelas.

A partir de los hallazgos de esta investigación, se propone incorporar a futuro al estudio de la política pública educativa, específicamente en el marco normativo en investigación: la Ley SEP, destinado a buscar mejoras a la educación chilena y equiparar la brecha de desigualdad de resultados, integrando nuevas correcciones 0 sugerencias técnicas y principalmente políticas que contribuyan a modificar estructuralmente el sistema, introduciendo cambios tanto en las asignaciones presupuestarias como en las formas de operar de la Ley, afectando principalmente la gestión escolar. Junto con ello, revisar los vínculos existentes entre este marco normativos y otros, los cuales tensionan el sistema educativo desde las altas exigencias requeridas exclusivamente en los resultados educativos estandarizados, dejando afectadas las comunidades escolares con menos posibilidades de ampliar su desarrollo en un proceso permanente de estancamiento.

El estudio hecho de la región de Valparaíso se ha realizado para aportar a la toma de decisiones a nivel de política pública nacional en educación. Las escuelas de la región no presentan cambios significativos en ocho años investigados (2008-2015), hecho que debiese considerarse para la presentación de propuestas que reorienten el fin último de esta política: mejorar la calidad de la educación en las escuelas que lo requieren.

\section{ReFREREIIIIS}

Agencia de Calidad. (2012). Informe técnico SIMCE 2012. Santiago de Chile. Recuperado de http://archivos.agenciaeducacion.cl/documentos-web/Informe_Tecnico_Simce_2012.pdf Agencia de Calidad. (2013). Informe técnico SIMCE 2013. Santiago de Chile. Recuperado de http://archivos.agenciaeducacion.cl/documentos-web/InformeTecnicoSimce_2013.pdf

Agencia de Calidad. (2014). Informe técnico SIMCE 2014. Santiago de Chile. Recuperado de http://archivos.agenciaeducacion.cl/InformeTecnicoSimce_2014.pdf

Amaya, M. (2015). Análisis de la Ley de Subvención Escolar Preferencial a escuelas vulnerables chilenas: una aproximación desde la teoría de la reproducción de Bourdieu. Revista Magíster. Elsevier. Recuperado de http:// www.elsevier.es/magister/

Bouveau, P. (2005). La discriminación positiva en el mundo. ¿Una utopía pedagógica? Política Educativa y Equidad, 49-58.

Brunner, J.J. y Ganga-Contreras, F. (2017). Vulnerabilidad educacional en América Latina: una aproximación desde la sociología de la educación con foco en la educación temprana. Opción, 33(84), 12-37. 
Carrasco, R., Pérez, M. y Núñez, D. (2015). Hacia una distribución más equitativa de las oportunidades educativas: ¿cuál es el impacto de la política de subvención preferencial en el desempeño académico de los alumnos más vulnerables en Chile? Pensamiento Educativo, $52(2)$.

DIPRES. (2009). Evaluación de programas nuevos (EPN). Chile: Programa de Subvención Escolar Preferencial (SEP) / Centro de Microdatos de la Universidad de Chile.

Donoso-Díaz, S., de Souza, Â.R. y Gouveia, A.B. (2016). Desafíos políticos claves para los sistemas escolares de Brasil y Chile. Revista Española de Educación Comparada, (27), 73.

Gallego, F. (2015). Actualización y diagnóstico del sistema escolar. En F. Gallego (ed.), Ideas en educación. Reflexiones y propuestas desde la UC (pp. 182-191). Chile. Ediciones Universidad Católica de Chile.

Harguindéguy, J.B. (2015). Análisis de políticas públicas. Biblioteca Universitaria de Editorial Tecnos.

Hernández, J., Cardona-Arango, D. y Segura-Cardona, Á.M. (2018). Construcción y análisis de un índice de vulnerabilidad social en la población joven. Revista Latinoamericana de Ciencias Sociales. Niñez y Juventud, 16(1), 403-412.

Hernández Sampieri, R., Fernández Collado, C. y Baptista Lucio, P. (2014). Metodología de la investigación ( $6^{\mathrm{a}}$ ed.). México: McGraw-Hill.

Hernández, M. y Raczynski, D. (2015). Elección de escuela en Chile: de las dinámicas de distinción y exclusión a la segregación socioeconómica del sistema escolar. Estudios Pedagógicos (Valdivia), 41(2), 127-141.

Junaeb. (2018): Bases de datos índice de vulnerabilidad. Recuperado de http://junaebabierta. junaeb.cl/catalogo-de-datos/

Levy, S. and Schady, N. (2013). Latin America's Social Policy. The Journal of Economic Perspectives, 27(2).

Ministerio de Desarrollo Social. (2018). Registro social de hogares. Subsecretaría de Evaluación Social, Banco Mundial.

Ministerio de Educación. (2008, enero 25). Ley Nº 20.248. Ley de subvención escolar preferencial. Diario Oficial República de Chile.

Ministerio de Educación. (2009, agosto 17). Ley Nº 20.307. Ley General de Educación. Diario Oficial República de Chile.

Ministerio de Educación. (2011, agosto 11). Ley No 20.529. Ley de Aseguramiento de la Calidad de la Educación. Diario Oficial República de Chile.

Ministerio de Educación. (2014). Decreto Supremo $N^{0} 73$. Estándares Indicativos de Desempeño para establecimientos educacionales. Chile: Unidad de Currículum y Evaluación.

Ministerio de Educación. (2018). Bases de datos para matrícula y subvención escolar preferencial. Recuperado de https://centroestudios.mineduc.cl/noticias/datos-abiertos/

Mizala, A. y Torche, F. (2013). ¿Logra la subvención escolar preferencial igualar los resultados educativos? Espacio Público, 9, 1-36.

Namakforoosh, M. (2000). El proceso de investigación. México: Prentice-Hall Hispanoamericana.

Ñanculeo Raguileo, M. y Merino Escobar, J. (2016). Una aproximación a la vulnerabilidad en el sistema de educación parvularia en Chile. Nóesis. Revista de Ciencias Sociales y Humanidades, 25(50), 51-88.

Parcerisa, L. y Falabella, A. (2017). La consolidación del Estado evaluador a través de políticas de rendición de cuentas: trayectoria, producción y tensiones en el sistema educativo chileno. Education Policy Analysis Archives/Archivos Analiticos de Politicas Educativas, (25), 1-24.

OCDE. (2002). Glosario de los principales términos sobre evaluación y gestión basada en resultados. Recuperado de https://www.oecd.org/dac/evaluation/2754804.pdf

OCDE. (2016). PISA 2015. Resultados claves. Mejores políticas para una vida mejor. Recuperado de https://search.proquest.com/openview/e8c066902afa5fla32b207d74370b87f/1?pqorigsite $=$ gscholar\&cbl $=2026456$ Recuperado de www.oecd.org/eco/surveys/economic-survey-chile.htm 
Pino-Yancovic, M., Oyarzún-Vargas, G. y Salinas-Barrios, I. (2016). Crítica a la rendición de cuentas: narrativa de resistencia al sistema de evaluación en chile. Cuadernos Cedes, 36(100).

Raczynski, D., Muñoz, G., Weinstein, J. y Pascual, J. (2013). Subvención escolar preferencial (SEP) en Chile: un intento por equilibrar la macro y micropolítica escolar. Reice. Revista Iberoamericana sobre Calidad, Eficacia y Cambio en Educación, 11(2), 164-193.

Rivas, A. y Sánchez, B. (2016). Políticas y resultados educativos en América Latina: un mapa comparado de siete países (2000-2015). Relieve. Revista Electrónica de Investigación y Evaluación Educativa, 22(1).

Venegas, S., Garay, M. y Sillard, M. (2016). La Ley SEP en las escuelas de la comuna de Punta Arenas, entre los años 2008 al 2015. Revista Sophia Austral, 95-109.

Weinstein, J., Fuenzalida, A. y Muñoz, G. (2010). La subvención preferencial: desde una difícil instalación hacia su institucionalización. Fin de Ciclo, 161-182. 
